\title{
Existence and Location Results for Fully Nonlinear Boundary Value Problem of nth-Order Nonlinear System
}

\author{
Li Sun, ${ }^{1}$ Mingru Zhou, ${ }^{1}$ and Guangwa Wang ${ }^{1,2}$ \\ ${ }^{1}$ Department of Mathematics, Xuzhou Normal University, Xuzhou 221116, China \\ ${ }^{2}$ Department of Mathematics, Suzhou University, Suzhou 215006, China \\ Correspondence should be addressed to Guangwa Wang, wanggw7653@163.com \\ Received 16 August 2008; Revised 10 December 2008; Accepted 21 January 2009 \\ Recommended by Ivan T. Kiguradze
}

By appropriate bounding function pair and modified functions, using the theory of differential inequalities, this paper presents the existence and location criteria of solutions for the system of general $n$ th-order differential equations with nonlinear boundary conditions. We give an example showing that the results are sharp. Our results extend many existing results.

Copyright $@ 2009 \mathrm{Li}$ Sun et al. This is an open access article distributed under the Creative Commons Attribution License, which permits unrestricted use, distribution, and reproduction in any medium, provided the original work is properly cited.

\section{Introduction}

In this paper, we are concerned with the following boundary value problem (BVP) for an $n$ th-order nonlinear system:

$$
\begin{gathered}
y^{(n)}=f\left(t, y, y^{\prime}, \ldots, y^{(n-1)}\right), \\
P_{i}\left(y(a), y^{\prime}(a), \ldots, y^{(n-1)}(a)\right)=0, \quad i=1, \ldots, n-1, \\
P_{n}\left(y(b), y^{\prime}(b), \ldots, y^{(n-1)}(b)\right)=0,
\end{gathered}
$$

where $t \in I=[a, b], y=\left(y_{1}, y_{2}, \ldots, y_{m}\right) \in \mathbb{R}^{m}, f\left(t, \xi_{0}, \xi_{1}, \ldots, \xi_{n-1}\right) \in C\left(I \times \mathbb{R}^{m n}, \mathbb{R}^{m}\right)$, $P_{i}\left(\eta_{0}, \eta_{1}, \ldots, \eta_{n-1}\right) \in C\left(\mathbb{R}^{m n}, \mathbb{R}^{m}\right), P_{n}\left(\zeta_{0}, \zeta_{1}, \ldots, \zeta_{n-1}\right) \in C\left(\mathbb{R}^{m n}, \mathbb{R}^{m}\right)$.

Boundary value problems for ordinary differential equations play a very important role in both theory and applications. They are used to describe a large number of physical, biological, and chemical phenomena. There have been many accomplishments on the study 
of the existence of solutions for BVPs of nonlinear differential equations using the theory of differential inequalities (cf. [1-31]). Although the method has been particularly fruitful for low-order ordinary differential equations (cf. [3-8, 14, 18, 31, 32]), Kelley [33] and Klaasen [3] did obtain early applications to higher-order ODEs. For more information, we refer the readers to $[2,3,9,10,12-16,19-21,26,28,33-35]$ and the references therein. For the case of differential systems, the results are few (cf. [2, 4-6, 8, 11, 29-32]). On the other hand, there are many papers, see $[17,18,22,23,25,36-39]$ and the references therein, concerning the existence of solutions for BVPs by using other approaches (e.g., the shooting method, many kinds of fixed-point theorems, many kinds of degree theories, etc.). However, there are very few results on the study of the existence of solutions for the general nonlinear system with the general nonlinear boundary conditions. To fill the gap, we will investigate BVP (1.1).

The aim of this paper is to generalize or complement the existing results. In order to do so, as a sequel of [28, 29], following the thoughts and methods of Fabry and Habets [1], the authors considered the nonlinear BVP (1.1) for differential equation and even the more general BVP (4.1) with the full nonlinear boundary conditions and obtained some results [40-43]. In this paper we consider the nonlinear BVP (1.1) for differential systems and even the more general BVP (4.1) with the full nonlinear boundary conditions. To the best of our knowledge, the general cases of BVP (1.1) and BVP (4.1) have not been studied in the available reference materials. By appropriate bounding function pair and modified functions, using the theory of differential inequalities, we establish some sufficient conditions which guarantee the existence of at least one solution for these BVPs. We give an example showing that our results are sharp.

A novel feature of our work is that we present a new definition of bounding function pair for BVP. It is well known that bounding function pair (i.e., upper-lower solutions in many references) is very important to study the existence of solutions for BVPs. Because of the complexity of the vector case (cf. [2, chapters 2 and 7]), how to give an appropriate definition of bounding function pair for the full nonlinear BVP of differential system is the difficulty in our work.

The method of this paper, which may be called simultaneous modification, is distinctive. It is not only modifying the nonlinear function in the original equations, but also transforming the original nonlinear boundary conditions into some new boundary conditions which are easy to discuss. Thus, we get the new BVP which will be discussed in the first place, then the judgement of the existence of solutions for the original BVP will be attained naturally. This technique dealing with the nonlinear problem is simpler and clearer compared with the method of shooting.

Throughout the paper, the comparison between the two vectors will be viewed as the same comparisons according to their components, and the operations between the two vectors will be viewed as the same operations according to their components.

The rest of this paper is organized as follows. In Section 2, we first give two basic definitions, that is, bounding function pair and the Nagumo condition, and then we study the modified boundary value problem of BVP (1.1), that is, BVP (2.17). Following the preparative theorem in Section 2, in Section 3, we state and prove the main result, that is, the sufficient criterion of the existence of solutions for BVP (1.1). In Section 4, a more general boundary value problem (4.1) is investigated. Moreover in Section 5, an example is illustrated to show that our results are sharp. Finally, in Section 6, some remarks are given. 


\section{Preparative Theorem}

\subsection{Basic Concepts}

We first define a function

$$
\delta(r, x, s) \equiv \begin{cases}r & \text { if } x<r \\ x & \text { if } r \leqslant x \leqslant s \\ s & \text { if } s<x\end{cases}
$$

where $r, x, s \in \mathbb{R}, r \leqslant s$. Moreover, if

$$
r=\left(r_{1}, \ldots, r_{m}\right), \quad x=\left(x_{1}, \ldots, x_{m}\right), \quad s=\left(s_{1}, \ldots, s_{m}\right),
$$

then, we define

$$
\delta(r, x, s) \equiv\left(\delta\left(r_{1}, x_{1}, s_{1}\right), \ldots, \delta\left(r_{m}, x_{m}, s_{m}\right)\right) .
$$

Definition 2.1. Assume that $\alpha, \beta \in C^{n}\left(I, \mathbb{R}^{m}\right)$. The pair of vector-valued functions $(\alpha(t), \beta(t))$ is called a bounding function pair (or simply, a bounding pair) of $\mathrm{BVP}(1.1)$ in case that for some positive constant $N$ depending on $(f, \alpha, \beta)$, and for all $u \in C^{n}\left(I, \mathbb{R}^{m}\right)$,

(i) $\alpha^{(i)}(t) \leqslant \beta^{(i)}(t), t \in I, i=0,1, \ldots, n-2$,

(ii)

$$
\begin{aligned}
& \alpha_{j}^{(n)}(t) \geqslant f_{j}\left(t, \bar{u}(t), \bar{u}^{\prime}(t), \ldots, \bar{u}^{(n-3)}(t), \bar{u}_{\alpha_{j}}^{(n-2)}(t), \bar{u}_{\alpha_{j}}^{(n-1)}(t)\right), \\
& \beta_{j}^{(n)}(t) \leqslant f_{j}\left(t, \bar{u}(t), \bar{u}^{\prime}(t), \ldots, \bar{u}^{(n-3)}(t), \bar{u}_{\beta_{j}}^{(n-2)}(t), \bar{u}_{\beta_{j}}^{(n-1)}(t)\right), \quad j=1, \ldots, m,
\end{aligned}
$$

where

$$
\begin{aligned}
\bar{u}^{(i)}(t) & =\delta\left(\alpha^{(i)}(t), u^{(i)}(t), \beta^{(i)}(t)\right), \quad i=0,1, \ldots, n-3, \\
\bar{u}_{\alpha_{j}}^{(k)}(t) & =\left(\bar{u}_{1}^{(k)}(t), \ldots, \bar{u}_{j-1}^{(k)}(t), \alpha_{j}^{(k)}(t), \bar{u}_{j+1}^{(k)}(t), \ldots, \bar{u}_{m}^{(k)}(t)\right), \\
\bar{u}_{\beta_{j}}^{(k)}(t) & =\left(\bar{u}_{1}^{(k)}(t), \ldots, \bar{u}_{j-1}^{(k)}(t), \beta_{j}^{(k)}(t), \bar{u}_{j+1}^{(k)}(t), \ldots, \bar{u}_{m}^{(k)}(t)\right), \quad k=n-2, n-1, \\
\bar{u}_{l}^{(n-2)}(t) & =\delta\left(\alpha_{l}^{(n-2)}(t), u_{l}^{(n-2)}(t), \beta_{l}^{(n-2)}(t)\right), \\
\bar{u}_{l}^{(n-1)} & =\delta\left(-N, u_{l}^{(n-1)}, N\right), \quad l=1,2, \ldots, j-1, j+1, \ldots, m ;
\end{aligned}
$$


(iii)

$$
\begin{aligned}
& P_{i, j}\left(\bar{u}(a), \ldots, \bar{u}^{(i-2)}(a), \bar{u}_{\alpha_{j}}^{(i-1)}(a), \bar{u}_{\alpha_{j}}^{(i)}(a), \bar{u}^{(i+1)}(a), \ldots, \bar{u}^{(n-1)}(a)\right) \\
& \quad \leqslant 0 \leqslant P_{i, j}\left(\bar{u}(a), \ldots, \bar{u}^{(i-2)}(a), \bar{u}_{\beta_{j}}^{(i-1)}(a), \bar{u}_{\beta_{j}}^{(i)}(a), \bar{u}^{(i+1)}(a), \ldots, \bar{u}^{(n-1)}(a)\right), \\
& P_{n, j}\left(\bar{u}(b), \ldots, \bar{u}^{(n-3)}(b), \bar{u}_{\alpha_{j}}^{(n-2)}(b), \bar{u}_{\alpha_{j}}^{(n-1)}(b)\right) \\
& \quad \leqslant 0 \leqslant P_{n, j}\left(\bar{u}(b), \ldots, \bar{u}^{(n-3)}(b), \bar{u}_{\beta_{j}}^{(n-2)}(b), \bar{u}_{\beta_{j}}^{(n-1)}(b)\right),
\end{aligned}
$$

where $i=1,2, \ldots, n-1, j=1,2, \ldots, m$, and

$$
\begin{aligned}
\bar{u}_{\alpha_{j}}^{(k)}(t) & =\left(\bar{u}_{1}^{(k)}(t), \ldots, \bar{u}_{j-1}^{(k)}(t), \alpha_{j}^{(k)}(t), \bar{u}_{j+1}^{(k)}(t), \ldots, \bar{u}_{m}^{(k)}(t)\right), \\
\bar{u}_{\beta_{j}}^{(k)}(t) & =\left(\bar{u}_{1}^{(k)}(t), \ldots, \bar{u}_{j-1}^{(k)}(t), \beta_{j}^{(k)}(t), \bar{u}_{j+1}^{(k)}(t), \ldots, \bar{u}_{m}^{(k)}(t)\right), \quad k=0,1,2, \ldots, n-1, \\
\bar{u}^{(n-2)}(t) & =\delta\left(\alpha^{(n-2)}(t), u^{(n-2)}(t), \beta^{(n-2)}(t)\right) \\
\bar{u}^{(n-1)}(a) & =\delta\left(-\bar{N}, u^{(n-1)}(a), \bar{N}\right), \quad \bar{N}=(N, \ldots, N) .
\end{aligned}
$$

Definition 2.2. A continuous function $f\left(t, \xi_{0}, \ldots, \xi_{n-1}\right)$ is said to satisfy the Nagumo condition with respect to variable $\xi_{n-1}$ on the set

$$
\begin{array}{r}
\mathfrak{D}=\left\{\left(t, \xi_{0}, \ldots, \xi_{n-1}\right)|t \in I ;| \xi_{j, k} \mid \leqslant r_{j}, j=0,1, \ldots, n-2,\right. \\
\left.k=1, \ldots, m, r_{j} \text { is a positive constant; } \xi_{n-1} \in \mathbb{R}^{m}\right\},
\end{array}
$$

in case there exist functions $\Phi_{i} \in C([0,+\infty),(0,+\infty)), i=1, \ldots, m$, such that

$$
\begin{gathered}
\left|f_{i}\left(t, \xi_{0}, \ldots, \xi_{n-1}\right)\right| \leqslant \Phi_{i}\left(\left|\xi_{n-1, i}\right|\right), \\
\int^{+\infty} \frac{s d s}{\Phi_{i}(s)}=+\infty .
\end{gathered}
$$

\subsection{The Modified Problem}

Assume that there are two vector-valued functions $\alpha(t), \beta(t)$ satisfying

$$
\alpha^{(j)}(t) \leqslant \beta^{(j)}(t), \quad j=0,1, \ldots, n-2 .
$$

We define function $\bar{f}\left(t, y, y^{\prime}, \ldots, y^{(n-1)}\right)$ which components are

$$
\bar{f}_{i}\left(t, y, y^{\prime}, \ldots, y^{(n-1)}\right) \equiv f_{i}\left(t, \bar{y}, \bar{y}^{\prime}, \ldots, \bar{y}^{(n-1)}\right)+h\left(y_{i}^{(n-2)}\right)
$$


where $\bar{y}^{(j)}(t)=\delta\left(\alpha^{(j)}(t), y^{(j)}(t), \beta^{(j)}(t)\right)(j=0,1, \ldots, n-2)$, and $\bar{y}^{(n-1)}(t)=\delta\left(-\bar{N}, y^{(n-1)}(t)\right.$, $\bar{N}), \bar{N}=(N, \ldots, N) . N$ is a positive constant such that

$$
\begin{gathered}
N>\max _{\substack{t \in I \\
i=1, \ldots, m}}\left\{\frac{2 M}{b-a},\left|\alpha_{i}^{(n-1)}(t)\right|,\left|\beta_{i}^{(n-1)}(t)\right|\right\}, \\
\int_{2 M /(b-a)}^{N} \frac{s d s}{\Phi_{i}(s)}>2 M
\end{gathered}
$$

in which $M>\max _{t \in I, i=1, \ldots, m}\left\{\left|\alpha_{i}^{(n-2)}(t)\right|,\left|\beta_{i}^{(n-2)}(t)\right|\right\} . h\left(y_{i}^{(n-2)}\right)$ is continuous, bounded, and

$$
h\left(y_{i}^{(n-2)}\right) \begin{cases}<0 & \text { if } y_{i}^{(n-2)}<\alpha_{i}^{(n-2)}, \\ =0 & \text { if } \alpha_{i}^{(n-2)} \leqslant y_{i}^{(n-2)} \leqslant \beta_{i}^{(n-2)}, \\ >0 & \text { if } y_{i}^{(n-2)}>\beta_{i}^{(n-2)}\end{cases}
$$

Such function $h(\cdot)$ is easy to obtain, for example, let

$$
h\left(y_{i}^{(n-2)}\right) \equiv \frac{y_{i}^{(n-2)}-\bar{y}_{i}^{(n-2)}}{1+\left|y_{i}^{(n-2)}-\bar{y}_{i}^{(n-2)}\right|}
$$

In addition, we define

$$
\begin{aligned}
& \bar{P}_{i}\left(y(t), y^{\prime}(t), \ldots, y^{(n-1)}(t)\right) \\
& \quad \equiv \delta\left(\alpha^{(i-1)}(t), y^{(i-1)}(t)-P_{i}\left(y(t), y^{\prime}(t), \ldots, y^{(n-1)}(t)\right), \beta^{(i-1)}(t)\right), \quad i=1,2, \ldots, n-1, \\
& \bar{P}_{n}\left(y(t), y^{\prime}(t), \ldots, y^{(n-1)}(t)\right) \\
& \quad \equiv \delta\left(\alpha^{(n-2)}(t), y^{(n-2)}(t)-P_{n}\left(y(t), y^{\prime}(t), \ldots, y^{(n-1)}(t)\right), \beta^{(n-2)}(t)\right) .
\end{aligned}
$$

Then, we consider the following modified problem:

$$
\begin{aligned}
y^{(n)} & =\bar{f}\left(t, y, y^{\prime}, \ldots, y^{(n-1)}\right), \\
y^{(i-1)}(a) & =\bar{P}_{i}\left(y(a), y^{\prime}(a), \ldots, y^{(n-1)}(a)\right), \quad i=1, \ldots, n-1, \\
y^{(n-2)}(b) & =\bar{P}_{n}\left(y(b), y^{\prime}(b), \ldots, y^{(n-1)}(b)\right) .
\end{aligned}
$$

\subsection{Preparative Theorem}

Lemma 2.3. Assume that

(A1) BVP (1.1) has a bounding pair $(\alpha(t), \beta(t))$ on the interval I by Definition 2.1; 
(A2) the function $f\left(t, \xi_{0}, \ldots, \xi_{n-1}\right)$ in BVP (1.1) satisfies the Nagumo condition with respect to $\xi_{n-1}$ by Definition 2.2.

Then, BVP (2.17) has a solution $y \in C^{n}\left(I, \mathbb{R}^{m}\right)$ such that

$$
\begin{gathered}
\alpha^{(i)}(t) \leqslant y^{(i)}(t) \leqslant \beta^{(i)}(t), \quad i=0,1, \ldots, n-2, \\
\left|y_{j}^{(n-1)}(t)\right| \leqslant N, \quad j=1,2, \ldots, m, t \in I
\end{gathered}
$$

where $N$ is defined in $\bar{f}_{i}$.

The following three propositions will lead to the proof of Lemma 2.3.

Proposition 2.4. The modified BVP (2.17) has a solution $y(t) \in C^{n}\left(I, \mathbb{R}^{m}\right)$.

Proof. Noticing that the functions $\bar{f}$ and $\overline{P_{i}}(i=1,2, \ldots, n)$ are bounded, this proposition immediately follows from the Schauder fixed-point theorem. The details here are omitted.

Proposition 2.5. Every solution $y(t)$ of the modified BVP (2.17) satisfies

$$
\alpha^{(i)}(t) \leqslant y^{(i)}(t) \leqslant \beta^{(i)}(t), \quad t \in I, i=0,1, \ldots, n-2
$$

Proof. First, we show that

$$
\alpha^{(n-2)}(t) \leqslant y^{(n-2)}(t) \leqslant \beta^{(n-2)}(t), \quad t \in I .
$$

If $\alpha^{(n-2)}(t) \leqslant y^{(n-2)}(t)$ is not true, then there exist some $i$ and $\xi \in[a, b]$ such that

$$
\max _{t \in I}\left(\alpha_{i}^{(n-2)}(t)-y_{i}^{(n-2)}(t)\right)=\alpha_{i}^{(n-2)}(\xi)-y_{i}^{(n-2)}(\xi)>0
$$

Then, $\xi \neq a, b$ by the boundary conditions of BVP (2.17). Thus,

$$
\begin{gathered}
\alpha_{i}^{(n-1)}(\xi)-y_{i}^{(n-1)}(\xi)=0, \\
\alpha_{i}^{(n)}(\xi)-y_{i}^{(n)}(\xi) \leqslant 0 .
\end{gathered}
$$

However, on the other hand, from the definition of $\alpha(t)$ and that $y(t)$ is a solution of (2.17), we have

$$
\begin{aligned}
\alpha_{i}^{(n)}(\xi)-y_{i}^{(n)}(\xi) \geqslant & f_{i}\left(\xi, \bar{y}(\xi), \ldots, \bar{y}^{(n-3)}(\xi), \bar{y}_{\alpha_{i}}^{(n-2)}(\xi), \bar{y}_{\alpha_{i}}^{(n-1)}(\xi)\right) \\
& -f_{i}\left(\xi, \bar{y}(\xi), \ldots, \bar{y}^{(n-3)}(\xi), \bar{y}^{(n-2)}(\xi), \bar{y}^{(n-1)}(\xi)\right)-h\left(y_{i}^{(n-2)}(\xi)\right) \\
= & -h\left(y_{i}^{(n-2)}(\xi)\right)>0 .
\end{aligned}
$$


This contradicts (2.23). Hence,

$$
\alpha^{(n-2)}(t) \leqslant y^{(n-2)}(t), \quad t \in I
$$

A similar proof shows that

$$
y^{(n-2)}(t) \leqslant \beta^{(n-2)}(t), \quad t \in I
$$

Summing up, (2.20) is true. From (2.20), the function $y^{(n-3)}(t)-\alpha^{(n-3)}(t)$ is increasing in $I$. Noticing

$$
\alpha^{(n-3)}(a) \leqslant y^{(n-3)}(a)
$$

we know that $\alpha^{(n-3)}(t) \leqslant y^{(n-3)}(t)$. A similar proof shows $y^{(n-3)}(t) \leqslant \beta^{(n-3)}(t)$. Using the same argument, it follows that $\alpha^{(i)}(t) \leqslant y^{(i)}(t) \leqslant \beta^{(i)}(t), i=n-4, n-5, \ldots, 2,1$. Thus, the proof of Proposition 2.5 is completed.

Proposition 2.6. Every solution $y(t)$ of the modified BVP (2.17) satisfies

$$
\left|y_{j}^{(n-1)}(t)\right| \leqslant N, \quad t \in I, j=1,2, \ldots, m
$$

Proof. Suppose that there exist some $j$ and $\tau \in[a, b]$ such that

$$
\left|y_{j}^{(n-1)}(\tau)\right|>N
$$

Without loss of generality, we assume that $y_{j}^{(n-1)}(\tau)>N$. There exists $\xi \in(a, b)$ such that

$$
y_{j}^{(n-1)}(\xi)=\frac{y_{j}^{(n-2)}(b)-y_{j}^{(n-2)}(a)}{b-a} \leqslant \frac{2 M}{b-a}<N .
$$

Hence, there exists some subinterval $[c, d]($ or $[d, c]) \subset[a, b]$ such that

$$
\begin{gathered}
y_{j}^{(n-1)}(c)=\frac{2 M}{b-a}, \quad y_{j}^{(n-1)}(d)=N, \\
\frac{2 M}{b-a} \leqslant y_{j}^{(n-1)}(t) \leqslant N, \quad \forall t \in[c, d](\text { or }[d, c]) .
\end{gathered}
$$


From condition (A2),

$$
\begin{aligned}
\left|\int_{c}^{d} \frac{y_{j}^{(n-1)}(s) y_{j}^{(n)}(s)}{\Phi_{j}\left(\left|y_{j}^{(n-1)}(s)\right|\right)} d s\right| & \leqslant\left|\int_{c}^{d} y_{j}^{(n-1)}(s) d s\right| \\
& =\left|y_{j}^{(n-2)}(d)-y_{j}^{(n-2)}(c)\right| \\
& \leqslant 2 M .
\end{aligned}
$$

On the other hand, from (2.13) we know that

$$
\begin{aligned}
\left|\int_{c}^{d} \frac{y_{j}^{(n-1)}(s) y_{j}^{(n)}(s)}{\Phi_{j}\left(\left|y_{j}^{(n-1)}(s)\right|\right)} d s\right| & =\left|\int_{2 M /(b-a)}^{N} \frac{r d r}{\Phi_{j}(r)}\right| \\
& =\int_{2 M /(b-a)}^{N} \frac{r d r}{\Phi_{j}(r)} \\
& >2 M .
\end{aligned}
$$

This inequality contradicts the above one and Proposition 2.6 holds.

The proof of Lemma 2.3 is now a simple consequence of Propositions 2.4, 2.5, and 2.6.

\section{Main Theorem}

Now, the main result of this paper is given in the following theorem.

Theorem 3.1. Let conditions (A1) and (A2) in Lemma 2.3 hold and assume that

(A3) the functions $P_{i, j}\left(\eta_{0}, \ldots, \eta_{n-1}\right)(i=1,2, \ldots, n-1, j=1,2, \ldots, m)$ are decreasing in $\eta_{i}$, while $P_{n, j}\left(\eta_{0}, \ldots, \eta_{n-1}\right)(j=1,2, \ldots, m)$ are increasing in $\eta_{n-1}$.

Then, BVP (1.1) has a solution $y \in C^{n}\left(I, \mathbb{R}^{m}\right)$ such that

$$
\begin{gathered}
\alpha^{(i)}(t) \leqslant y^{(i)}(t) \leqslant \beta^{(i)}(t), \quad i=0,1, \ldots, n-2, \\
\left|y_{j}^{(n-1)}(t)\right| \leqslant N, \quad j=1,2, \ldots, m, t \in I,
\end{gathered}
$$

where $N$ is defined in $\bar{f}_{i}$.

Proof. From Lemma 2.3 and the definition of $\bar{f}$, the solution $y(t)$ of the modified BVP (2.17) satisfies (1.1). Obviously, if it is proved that $y(t)$ satisfies the boundary conditions of (1.1) under condition (A3), we may conclude that $y(t)$ is just the solution of BVP (1.1).

First, we prove that

$$
P_{i}\left(y(a), \ldots, y^{(n-1)}(a)\right)=0, \quad i=1,2, \ldots, n-1 .
$$


Case 1. Suppose that

$$
\alpha^{(i-1)}(a) \leqslant y^{(i-1)}(a)-P_{i}\left(y(a), \ldots, y^{(n-1)}(a)\right) \leqslant \beta^{(i-1)}(a)
$$

Then

$$
\begin{aligned}
y^{(i-1)}(a) & =\bar{P}_{i}\left(y(a), \ldots, y^{(n-1)}(a)\right) \\
& =y^{(i-1)}(a)-P_{i}\left(y(a), \ldots, y^{(n-1)}(a)\right)
\end{aligned}
$$

Thus,

$$
P_{i}\left(y(a), \ldots, y^{(n-1)}(a)\right)=0 .
$$

Case 2. Suppose that there exist some $i \in\{1,2, \ldots, n-1\}$ and some $j \in\{1,2, \ldots, m\}$ such that

$$
\alpha_{j}^{(i-1)}(a)>y_{j}^{(i-1)}(a)-P_{i, j}\left(y(a), y^{\prime}(a), \ldots, y^{(n-1)}(a)\right)
$$

Then,

$$
y_{j}^{(i-1)}(a)=\bar{P}_{i, j}\left(y(a), y^{\prime}(a), \ldots, y^{(n-1)}(a)\right)=\alpha_{j}^{(i-1)}(a) .
$$

Hence,

$$
P_{i, j}\left(y(a), y^{\prime}(a), \ldots, y^{(n-1)}(a)\right)>0
$$

From Proposition 2.5,

$$
\bar{y}^{(k)}(a)=\delta\left(\alpha^{(k)}(a), y^{(k)}(a), \beta^{(k)}(a)\right)=y^{(k)}(a), \quad k=0,1, \ldots, n-2
$$

From Proposition 2.6,

$$
\bar{y}^{(n-1)}(a)=\delta\left(-\bar{N}, y^{(n-1)}(a), \bar{N}\right)=y^{(n-1)}(a) .
$$

When $i=1,2, \ldots, n-2$, Proposition 2.5 implies

$$
\bar{y}_{\alpha_{j}}^{(i)}(a) \leqslant y^{(i)}(a), \quad j=1,2, \ldots, m
$$

When $i=n-1$, formula (3.7) implies

$$
\bar{y}_{j}^{(n-2)}(a)=\alpha_{j}^{(n-2)}(a), \quad j=1,2, \ldots, m .
$$


Recalling that $\bar{y}_{j}^{(n-2)}(t) \geqslant \alpha_{j}^{(n-2)}(t), t \in[a, b]$, we get $\bar{y}_{j}^{(n-1)}(a) \geqslant \alpha_{j}^{(n-1)}(a)$. Thus,

$$
\bar{y}_{\alpha_{j}}^{(n-1)}(a) \leqslant y^{(n-1)}(a), \quad j=1,2, \ldots, m .
$$

It follows from formulas (3.7)-(3.13) and condition (A3) that, for $i=1,2, \ldots, n-1$,

$$
\begin{aligned}
& P_{i, j}\left(\bar{y}(a), \ldots, \bar{y}^{(i-2)}(a), \bar{y}_{\alpha_{j}}^{(i-1)}(a), \bar{y}_{\alpha_{j}}^{(i)}(a), \bar{y}^{(i+1)}(a), \ldots, \bar{y}^{(n-1)}(a)\right) \\
& \quad=P_{i, j}\left(\bar{y}(a), \ldots, \bar{y}^{(i-2)}(a), y^{(i-1)}(a), \bar{y}_{\alpha_{j}}^{(i)}(a), \bar{y}^{(i+1)}(a), \ldots, \bar{y}^{(n-1)}(a)\right) \\
& \quad \geqslant P_{i, j}\left(\bar{y}(a), \ldots, \bar{y}^{(i-2)}(a), y^{(i-1)}(a), y^{(i)}(a), \bar{y}^{(i+1)}(a), \ldots, \bar{y}^{(n-1)}(a)\right)>0 .
\end{aligned}
$$

It is easy to see that the last inequality contradicts (iii) of Definition 2.1. Therefore, Case 2 is not true.

Case 3. Suppose that there exist some $i \in\{1,2, \ldots, n-1\}$ and some $j \in\{1,2, \ldots, m\}$ such that

$$
y_{j}^{(i-1)}(a)-P_{i, j}\left(y(a), y^{\prime}(a), \ldots, y^{(n-1)}(a)\right)>\beta_{j}^{(i-1)}(a) .
$$

Then,

$$
y_{j}^{(i-1)}(a)=\bar{P}_{i, j}\left(y(a), y^{\prime}(a), \ldots, y^{(n-1)}(a)\right)=\beta_{j}^{(i-1)}(a)
$$

Hence,

$$
P_{i, j}\left(y(a), y^{\prime}(a), \ldots, y^{(n-1)}(a)\right)<0
$$

Similar to the argument of Case 2, we have

$$
\begin{aligned}
& P_{i, j}\left(\bar{y}(a), \ldots, \bar{y}^{(i-2)}(a), \bar{y}_{\beta_{j}}^{(i-1)}(a), \bar{y}_{\beta_{j}}^{(i)}(a), \bar{y}^{(i+1)}(a), \ldots, \bar{y}^{(n-1)}(a)\right) \\
& \quad=P_{i, j}\left(\bar{y}(a), \ldots, \bar{y}^{(i-2)}(a), y^{(i-1)}(a), \bar{y}_{\beta_{j}}^{(i)}(a), \bar{y}^{(i+1)}(a), \ldots, \bar{y}^{(n-1)}(a)\right) \\
& \quad \leqslant P_{i, j}\left(\bar{y}(a), \ldots, \bar{y}^{(i-2)}(a), y^{(i-1)}(a), y^{(i)}(a), \bar{y}^{(i+1)}(a), \ldots, \bar{y}^{(n-1)}(a)\right)<0 .
\end{aligned}
$$

Obviously, the last inequality contradicts (iii) of Definition 2.1. Therefore, this case cannot hold. Summing up, (3.2) holds.

A similar proof shows that

$$
P_{n}\left(y(b), y^{\prime}(b), \ldots, y^{(n-1)}(b)\right)=0 .
$$

Consequently, the proof is completed. 
Remark 3.2. From (iii) of Definition 2.1 and (A3) of Theorem 3.1, it is easy to see that the functions $P_{i, j}\left(\eta_{0}, \ldots, \eta_{n-1}\right)(i=1,2, \ldots, n-1, j=1,2, \ldots, m)$ should be increasing in $\eta_{i-1}$.

\section{A Generalized Problem}

Now, we consider the following boundary value problem with more generalized boundary conditions:

$$
\begin{gathered}
y^{(n)}=f\left(t, y, \ldots, y^{(n-1)}\right) \\
P_{i}\left(y(a), \ldots, y^{(n-1)}(a), y(b), \ldots, y^{(n-1)}(b)\right)=0,
\end{gathered}
$$

where $t \in I, i=1,2, \ldots, n, y \in \mathbb{R}^{m}, f$ and $P_{i}$ are continuous $m$-dimensional vector-valued functions.

Similar to Definition 2.1, we give the following.

Definition 4.1. Assume $\alpha, \beta \in C^{n}\left(I, \mathbb{R}^{m}\right)$, The pair of vector-valued functions $(\alpha(t), \beta(t))$ is called a bounding function pair of BVP (4.1) in case that

(i) same as (i) of Definition 2.1;

(ii) same as (ii) of Definition 2.1;

$(\text { (iii) })^{\prime}$

$$
\begin{aligned}
& P_{i, j}\left(\bar{u}(a), \ldots, \bar{u}_{\alpha_{j}}^{(i-1)}(a), \bar{u}_{\alpha_{j}}^{(i)}(a), \ldots, \bar{u}^{(n-1)}(a), \bar{u}(b), \ldots, \bar{u}^{(n-1)}(b)\right) \\
& \quad \leqslant 0 \leqslant P_{i, j}\left(\bar{u}(a), \ldots, \bar{u}_{\beta_{j}}^{(i-1)}(a), \bar{u}_{\beta_{j}}^{(i)}(a), \ldots, \bar{u}^{(n-1)}(a), \bar{u}(b), \ldots, \bar{u}^{(n-1)}(b)\right), \\
& P_{n, j}\left(\bar{u}(a), \ldots, \bar{u}^{(n-1)}(a), \bar{u}(b), \ldots, \bar{u}^{(n-3)}(b), \bar{u}_{\alpha_{j}}^{(n-2)}(b), \bar{u}_{\alpha_{j}}^{(n-1)}(b)\right) \\
& \quad \leqslant 0 \leqslant P_{n, j}\left(\bar{u}(a), \ldots, \bar{u}^{(n-1)}(a), \bar{u}(b), \ldots, \bar{u}^{(n-3)}(b), \bar{u}_{\beta_{j}}^{(n-2)}(b), \bar{u}_{\beta_{j}}^{(n-1)}(b)\right),
\end{aligned}
$$

where $i=1,2, \ldots, n-1 ; j=1,2, \ldots, m$.

For BVP (4.1), we have the following existence theorem.

Theorem 4.2. Assume that

(A1)' BVP (4.1) has a bounding function pair $(\alpha(t), \beta(t))$ in the interval I by Definition 4.1;

(A2)' the function $f\left(t, \xi_{0}, \ldots, \xi_{n-1}\right)$ in BVP (4.1) satisfies the Nagumo condition with respect to $\xi_{n-1}$ by Definition 2.2;

(A3)' the functions $P_{i, j}\left(\eta_{0}, \ldots, \eta_{n-1}, \zeta_{0}, \ldots, \zeta_{n-1}\right)(i=1,2, \ldots, n-1, j=1,2, \ldots, m)$ are decreasing in $\eta_{i}$, while $P_{n, j}\left(\eta_{0}, \ldots, \eta_{n-1}, \zeta_{0}, \ldots, \zeta_{n-1}\right)(j=1,2, \ldots, m)$ are increasing in $\eta_{n-1}$. 
Then, BVP (4.1) has a solution $y \in C^{n}\left(I, \mathbb{R}^{m}\right)$ such that

$$
\begin{gathered}
\alpha^{(i)}(t) \leqslant y^{(i)}(t) \leqslant \beta^{(i)}(t), \quad i=0,1, \ldots, n-2, \\
\left|y_{j}^{(n-1)}(t)\right| \leqslant N, \quad j=1,2, \ldots, m, t \in I,
\end{gathered}
$$

where $N$ is defined in $\bar{f}_{i}$.

Proof. Consider the modified problem

$$
\begin{gathered}
y^{(n)}=\bar{f}\left(t, y, \ldots, y^{(n-1)}\right), \\
y^{(i-1)}(a)=\bar{P}_{i}(a), \quad i=1,2, \ldots, n-1, \\
y^{(n-2)}(b)=\bar{P}_{n}(b) .
\end{gathered}
$$

The modified function $\bar{f}\left(t, y, \ldots, y^{(n-1)}\right)$ is defined as BVP (2.17), and

$$
\begin{gathered}
\bar{P}_{i}(t) \equiv \bar{P}_{i}\left(y(t), \ldots, y^{(n-1)}(t), y(b+a-t), \ldots, y^{(n-1)}(b+a-t)\right) \\
\equiv \delta\left(\alpha^{(i-1)}(t), y^{(i-1)}(t)-P_{i}\left(y(t), \ldots, y^{(n-1)}(t), y(b+a-t), \ldots,\right.\right. \\
\left.\left.y^{(n-1)}(b+a-t)\right), \beta^{(i-1)}(t)\right)
\end{gathered}
$$

where $i=1,2, \ldots, n-1$,

$$
\begin{aligned}
\bar{P}_{n}(t) \equiv & \bar{P}_{n}\left(y(b+a-t), \ldots, y^{(n-1)}(b+a-t), y(t), \ldots, y^{(n-1)}(t)\right) \\
\equiv & \delta\left(\alpha^{(n-2)}(t), y^{(n-2)}(t)-P_{n}\left(y(b+a-t), \ldots, y^{(n-1)}(b+a-t), y(t), \ldots,\right.\right. \\
& \left.\left.\quad y^{(n-1)}(t)\right), \beta^{(n-2)}(t)\right) .
\end{aligned}
$$

Using the same argument as the proof of Lemma 2.3, it follows from conditions (A1)' and $(\mathrm{A} 2)^{\prime}$ that BVP (4.4) has a solution $y(t)$ satisfying the two inequalities in the conclusions of Lemma 2.3. Furthermore, in an analogous way to the proof of Theorem 3.1, it follows that the solution $y(t)$ of BVP(4.4) is just a solution of BVP (4.1). Consequently, the proof of Theorem 4.2 is completed. The details of the proof will be omitted.

Remark 4.3. From (iii) ${ }^{\prime}$ of Definition 4.1 and (A3)' of Theorem 4.2, it is easy to see that the functions $P_{i, j}\left(\eta_{0}, \ldots, \eta_{n-1}, \zeta_{0}, \ldots, \zeta_{n-1}\right)(i=1,2, \ldots, n-1, j=1,2, \ldots, m)$ should be increasing in $\eta_{i-1}$. 


\section{An Example}

In this section, we present an example by making use of Theorems 3.1 and 4.2. With the example, we try to illustrate the applicability of our results and techniques and show that a bounding pair according to Definitions 2.1 or 4.1 can exist naturally.

Example 5.1. Consider the following 4th-order nonlinear system:

$$
\begin{aligned}
& y_{1}^{(4)}=\frac{1}{27} y_{1}^{3}+\frac{1}{2}\left(y_{2}^{\prime}\right)^{2}+\left(\frac{y_{1}^{\prime \prime}}{3}\right)^{3}+\left(y_{1}^{\prime}\right)^{2} \sin \left(y_{2}^{\prime \prime \prime}\right)^{2}+\sin t+4 \\
& y_{2}^{(4)}=y_{2}^{2}+\frac{1}{2}\left(y_{1}^{\prime}\right)^{2}+\left(\frac{y_{2}^{\prime \prime}}{5}\right)^{3}+\frac{1}{144}\left(y_{2}^{\prime \prime \prime}\right)^{2} \sin \left(y_{1}^{\prime \prime \prime}\right)^{2}+t^{2}
\end{aligned}
$$

together with the following boundary conditions:

$$
\begin{aligned}
& P_{1}:\left\{\begin{array}{l}
y_{1}(0)-e^{\left(y_{1}^{\prime}(0)\right)^{3}}=2.6, \\
y_{2}(0)+\frac{k}{36}\left(y_{2}^{\prime}(1)\right)^{2}=3.5,
\end{array}\right. \\
& P_{2}:\left\{\begin{array}{l}
2 y_{1}^{\prime}(0)-3\left(y_{1}^{\prime \prime}(0)\right)^{5}=0, \\
y_{1}(0)+\frac{1}{y_{2}^{\prime}(0)-1}+k\left(y_{1}^{\prime \prime}(1)\right)^{2}=1.6,
\end{array}\right. \\
& P_{3}:\left\{\begin{array}{l}
y_{1}^{\prime \prime}(0)+y_{2}^{\prime}(0)=0, \\
k\left(y_{2}(1)\right)^{2}-2\left(y_{2}^{\prime \prime}(0)\right)^{4}+k y_{2}^{\prime \prime}(1)=-3,
\end{array}\right. \\
& P_{4}:\left\{\begin{array}{l}
y_{1}^{\prime \prime}(1)=0, \\
k y_{1}(0)+y_{2}^{\prime}(1)+6 y_{2}^{\prime \prime}(1)+\frac{1}{12^{3}}\left(y_{2}^{\prime \prime \prime}(1)\right)^{3}=-2,
\end{array}\right.
\end{aligned}
$$

where $t \in[0,1]$, and $k$ is a constant.

Let

$$
\alpha(t)=\left(\begin{array}{c}
t^{4}-2 t^{3}+2.6 \\
t^{4}-2 t^{3}-t^{2}+3
\end{array}\right), \quad \beta(t)=\left(\begin{array}{c}
2.6 \\
4
\end{array}\right), \quad t \in[0,1]
$$

Then, for the case of $k=0$ and the case of $k=1$, by direct calculation, it is easy to check that $(\alpha(t), \beta(t))$ is a bounding pair of BVP (5.1) and all assumptions of Theorems 3.1 and 4.2 are 
fulfilled, respectively. Hence, for any of the two cases, BVP (5.1) has at least one solution $y(t)$ satisfying

$$
\begin{gathered}
\left(\begin{array}{c}
t^{4}-2 t^{3}+2.6 \\
t^{4}-2 t^{3}-t^{2}+3
\end{array}\right) \leqslant y(t) \leqslant\left(\begin{array}{c}
2.6 \\
4
\end{array}\right), \\
\left(\begin{array}{c}
4 t^{3}-6 t^{2} \\
4 t^{3}-6 t^{2}-2 t
\end{array}\right) \leqslant y^{\prime}(t) \leqslant\left(\begin{array}{l}
0 \\
0
\end{array}\right), \\
\left(\begin{array}{c}
12 t^{2}-12 t \\
12 t^{2}-12 t-t
\end{array}\right) \leqslant y^{\prime \prime}(t) \leqslant\left(\begin{array}{l}
0 \\
0
\end{array}\right),
\end{gathered}
$$

for each $t \in[0,1]$.

\section{Remarks}

(1) If the directions of the signs of inequalities in condition (iii) of Definition 2.1 are all changed to the opposite, and conditions (i), (ii) of Definition 2.1 hold, then we denote the revised definition by Definition 2.1. We obtain the following theorem similar to Theorem 3.1.

Theorem 6.1. Assume that

(A1)" BVP (1.1) has a bounding function pair $(\alpha(t), \beta(t))$ by Definition 6.1;

(A2) same as (A2) of Theorem 3.1;

(A3)" the monotony of $P_{i, j}$ is opposite to that of $(A 3)$.

Then, the conclusion of Theorem 3.1 still holds.

In fact, if we replace $P_{i}(i=1,2, \ldots, n)$ by $-P_{i}$ in Theorem 3.1, then, it follows from Theorem 3.1 that Theorem 6.1 is true. We may make the analogous argument for BVP (4.1).

(2) The essentiality of the modified function is to modify a general nonlinear continuous function to a continuous bounded function. It was appearing in different forms in references. In this paper, we give out one concise form.

(3) The definitions of scalar bounding functions are a good many. In this paper, the definitions in vector cases given are new and can be regarded as a kind of improvement and generalization. Of course, the conditions of the definitions may be changed by the actual need. For example, we take Definition 2.1 to discuss the following.

If $\bar{u}_{\alpha_{i}}^{(n-1)}(t), \bar{u}_{\beta_{i}}^{(n-1)}(t)$ in $f_{i}$ of condition (ii) are changed to $\bar{u}^{(n-1)}(t)$, respectively, we still may assure that those results hold.

If both $\bar{u}_{\alpha_{i}}^{(n-2)}$ and $\bar{u}_{\beta_{i}}^{(n-2)}(t)$ in $f_{i}$ of condition (ii) are modified to $\bar{u}^{(n-2)}(t)$, we may simplify the depiction and the proof. But, the modified condition becomes stronger.

It should be pointed out that condition (ii) may be weakened as

$$
\begin{aligned}
& \alpha_{i}^{(n)}(t) \geqslant f_{i}\left(t, \alpha(t), \alpha^{\prime}(t), \ldots, \alpha^{(n-2)}(t), \bar{u}^{(n-1)}(t)\right), \\
& \beta_{i}^{(n)}(t) \leqslant f_{i}\left(t, \beta(t), \beta^{\prime}(t), \ldots, \beta^{(n-2)}(t), \bar{u}^{(n-1)}(t)\right) .
\end{aligned}
$$


However, when proving Proposition 2.5, we should add one condition " $f_{i}$ are all decreasing in $\xi_{0}, \xi_{1}, \ldots, \xi_{n-2}$ ". Thus, $(*)$ implies condition (ii) of Definition 2.1. Consequently, condition (ii) about $\alpha, \beta$ seems weaker, but in fact, the whole requirement becomes stronger in some sense.

We also may discuss condition (iii) of Definition 2.1 in a similar way.

(4) The Nagumo condition in this paper ensures that the integral inequality (2.13) is true and essentially ensures that the derivative functions of solutions of the considered problems are bounded. Indeed, in some references, the integral equality

$$
\int^{+\infty} \frac{s d s}{\Phi_{i}(s)}=+\infty
$$

is straightly substituted by inequality (2.13). Moreover, we exhibit some new forms of the integral inequality (see [29]).

(5) From Theorems 3.1, 4.2, and the above remarks, we include or improve the results in [1-43], since our system and boundary conditions are fully nonlinear. Obviously, the results in all the references are not available to our example.

(6) Last but not least, it should be pointed out that although this paper presents the existence and location criteria of solutions for BVPs, the premise is that the bounding function pair is assumed to be existing. It is well known how to get a precise bounding function pair for a given BVP is a very difficult job in the theory of upper-lower solutions and remains unsolved.

\section{Acknowledgments}

The authors would like to express their gratitude to the reviewers for their careful reading of the manuscript and for their very helpful comments. This paper is supported by Science Research Innovation Project for Graduates of Jiangsu Province (CX07B-029Z), Natural Science Foundation of XZNU (08XLB03) and Qing Lan Project of XZNU.

\section{References}

[1] Ch. Fabry and P. Habets, "Upper and lower solutions for second-order boundary value problems with nonlinear boundary conditions," Nonlinear Analysis: Theory, Methods \& Applications, vol. 10, no. 10, pp. 985-1007, 1986.

[2] K. W. Chang and F. A. Howes, Nonlinear Singular Perturbation Phenomena: Theory and Applications, vol. 56 of Applied Mathematical Sciences, Springer, New York, NY, USA, 1984.

[3] G. A. Klaasen, "Differential inequalities and existence theorems for second and third order boundary value problems," Journal of Differential Equations, vol. 10, no. 3, pp. 529-537, 1971.

[4] F. A. Howes, "Differential inequalities and applications to nonlinear singular perturbation problems," Journal of Differential Equations, vol. 20, no. 1, pp. 133-149, 1976.

[5] W. G. Kelley, "A geometric method of studying two point boundary value problems for second order systems," The Rocky Mountain Journal of Mathematics, vol. 7, no. 2, pp. 251-263, 1977.

[6] W. G. Kelley, "Second order systems with nonlinear boundary conditions," Proceedings of the American Mathematical Society, vol. 62, no. 2, pp. 287-292, 1977.

[7] L. H. Erbe, "Existence of solutions to boundary value problems for second order differential equations," Nonlinear Analysis: Theory, Methods \& Applications, vol. 6, no. 11, pp. 1155-1162, 1982.

[8] M. A. O'Donnell, "Boundary and corner layer behavior in singularly perturbed semilinear systems of boundary value problems," SIAM Journal on Mathematical Analysis, vol. 15, no. 2, pp. 317-332, 1984. 
[9] A. Cabada, "The method of lower and upper solutions for $n$ th-order periodic boundary value problems," Journal of Applied Mathematics and Stochastic Analysis, vol. 7, no. 1, pp. 33-47, 1994.

[10] A. Cabada, "The method of lower and upper solutions for second, third, fourth, and higher order boundary value problems," Journal of Mathematical Analysis and Applications, vol. 185, no. 2, pp. 302320, 1994.

[11] V. F. Butuzov, N. N. Nefedov, and K. R. Schneider, "Singularly perturbed boundary value problems for systems of Tichonov's type in case of exchange of stabilities," Journal of Differential Equations, vol. 159, no. 2, pp. 427-446, 1999.

[12] A. Cabada, M. do R. Grossinho, and F. Minhós, “On the solvability of some discontinuous third order nonlinear differential equations with two point boundary conditions," Journal of Mathematical Analysis and Applications, vol. 285, no. 1, pp. 174-190, 2003.

[13] A. Cabada and E. Liz, "Boundary value problems for higher order ordinary differential equations with impulses," Nonlinear Analysis: Theory, Methods E Applications, vol. 32, no. 6, pp. 775-786, 1998.

[14] A. Cabada and F. M. Minhós, "Fully nonlinear fourth-order equations with functional boundary conditions," Journal of Mathematical Analysis and Applications, vol. 340, no. 1, pp. 239-251, 2008.

[15] Z. Du, W. Ge, and X. Lin, "Existence of solutions for a class of third-order nonlinear boundary value problems," Journal of Mathematical Analysis and Applications, vol. 294, no. 1, pp. 104-112, 2004.

[16] J. Ehme, P. W. Eloe, and J. Henderson, "Existence of solutions for $2 n$th order nonlinear generalized Sturm-Liouville boundary value problems," Mathematical Inequalities \& Applications, vol. 4, no. 2, pp. 247-255, 2001.

[17] J. Ehme, "Uniqueness and existence for perturbed focal boundary value problems," Applied Mathematics Letters, vol. 18, no. 8, pp. 875-879, 2005.

[18] J. Ehme and A. Lanz, "Uniqueness and existence for bounded boundary value problems," Journal of Mathematical Analysis and Applications, vol. 319, no. 2, pp. 725-731, 2006.

[19] F. Sadyrbaev, "Nonlinear fourth-order two-point boundary value problems," The Rocky Mountain Journal of Mathematics, vol. 25, no. 2, pp. 757-781, 1995.

[20] D. Franco, D. O’Regan, and J. Perán, "Fourth-order problems with nonlinear boundary conditions," Journal of Computational and Applied Mathematics, vol. 174, no. 2, pp. 315-327, 2005.

[21] D. Guo, "Extremal solutions for $n$ th-order impulsive integro-differential equations on the half-line in Banach spaces," Nonlinear Analysis: Theory, Methods E Applications, vol. 65, no. 3, pp. 677-696, 2006.

[22] Y. Li, "Existence and uniqueness for higher order periodic boundary value problems under spectral separation conditions," Journal of Mathematical Analysis and Applications, vol. 322, no. 2, pp. 530-539, 2006.

[23] Y. Liu, P. Yang, and W. Ge, "Solutions of two-point BVPs at resonance for higher order impulsive differential equations," Nonlinear Analysis: Theory, Methods \& Applications, vol. 60, no. 5, pp. 887-923, 2005.

[24] M. El-Shahed, "Positive solutions of boundary value problems for $n$th order ordinary differential equations," Electronic Journal of Qualitative Theory of Differential Equations, no. 1, pp. 1-9, 2008.

[25] C. Pang, W. Dong, and Z. Wei, "Green's function and positive solutions of $n$th order $m$-point boundary value problem," Applied Mathematics and Computation, vol. 182, no. 2, pp. 1231-1239, 2006.

[26] V. Šeda, J. J. Nieto, and M. Gera, "Periodic boundary value problems for nonlinear higher order ordinary differential equations," Applied Mathematics and Computation, vol. 48, no. 1, pp. 71-82, 1992.

[27] B. Yang, "Estimates of positive solutions for higher order right focal boundary value problem," Communications in Mathematical Analysis, vol. 4, no. 1, pp. 1-9, 2008.

[28] G. W. Wang and L. Sun, "Existence of solutions of nonlinear two-point boundary value problems for fourth-order differential equations," Journal of Xuzhou Normal University, vol. 19, no. 2, pp. 1-5, 2001.

[29] G. W. Wang, M. R. Zhou, and L. Sun, "Existence of solutions of two-point boundary value problems for the systems of $n$ th-order differential equations," Journal of Nanjing University. Mathematical Biquarterly, vol. 19, no. 1, pp. 68-79, 2002.

[30] X. Yang, "Existence of positive solutions for $2 m$-order nonlinear differential systems," Nonlinear Analysis: Theory, Methods E Applications, vol. 61, no. 1-2, pp. 77-95, 2005.

[31] X. Yang, "The method of lower and upper solutions for systems of boundary value problems," Applied Mathematics and Computation, vol. 144, no. 1, pp. 169-172, 2003.

[32] A. Lasota and J. A. Yorke, “Existence of solutions of two-point boundary value problems for nonlinear systems," Journal of Differential Equations, vol. 11, pp. 509-518, 1972.

[33] W. G. Kelley, "Some existence theorems for $n$ th-order boundary value problems," Journal of Differential Equations, vol. 18, no. 1, pp. 158-169, 1975. 
[34] J. Ehme, P. W. Eloe, and J. Henderson, "Upper and lower solution methods for fully nonlinear boundary value problems," Journal of Differential Equations, vol. 180, no. 1, pp. 51-64, 2002.

[35] Z. Du, W. Ge, and M. Zhou, "Singular perturbations for third-order nonlinear multi-point boundary value problem," Journal of Differential Equations, vol. 218, no. 1, pp. 69-90, 2005.

[36] R. P. Agarwal and I. Kiguradze, "Two-point boundary value problems for higher-order linear differential equations with strong singularities," Boundary Value Problems, vol. 2006, Article ID 83910, 32 pages, 2006.

[37] R. P. Agarwal and P. J. Y. Wong, "Existence of solutions for singular boundary problems for higher order differential equations," Rendiconti del Seminario Matematico e Fisico di Milano, vol. 65, no. 1, pp. 249-264, 1995.

[38] I. Kiguradze, "On periodic solutions of $n$th order ordinary differential equations," Nonlinear Analysis: Theory, Methods E Applications, vol. 40, no. 1-8, pp. 309-321, 2000.

[39] I. T. Kiguradze and T. Kusano, "On periodic solutions of higher-order nonautonomous ordinary differential equations," Differential Equations, vol. 35, no. 1, pp. 70-77, 1999.

[40] G. Wang, M. Zhou, and L. Sun, "Bounding functions methods for fully nonlinear boundary value problems," Nonlinear Analysis: Theory, Methods E Applications, vol. 64, no. 4, pp. 696-705, 2006.

[41] G. Wang, M. Zhou, and L. Sun, "Differential inequalities method to $n$ th-order boundary value problems," Journal of Inequalities and Applications, vol. 2006, Article ID 12040, 12 pages, 2006.

[42] G. Wang, M. Zhou, and L. Sun, "Existence of solutions of boundary value problem for 3rd order nonlinear system," Applied Mathematics and Computation, vol. 189, no. 2, pp. 1131-1138, 2007.

[43] G. Wang, M. Zhou, and L. Sun, "Fourth-order problems with fully nonlinear boundary conditions," Journal of Mathematical Analysis and Applications, vol. 325, no. 1, pp. 130-140, 2007. 